\title{
Antibioticoprofilaxia em cirurgias de cães e gatos: necessidade e realidade ${ }^{1}$
}

\author{
Daniel Pontes Braga², Andréa Pacheco Batista Borges ${ }^{3}$, Tatiana Borges de Carvalho ${ }^{4}$, \\ Letícia Corrêa Santos ${ }^{5}$, Camila Maria Mantovani Corsini ${ }^{5}$
}

\begin{abstract}
RESUMO
A administração profilática de antimicrobianos tem por objetivo evitar a contaminação do ferimento cirúrgico, após exposição a algum micro-organismo e antes da instalação do processo de infecção, devendo alcançar e manter concentrações antimicrobianas inibitórias, no local da incisão, durante todo o procedimento cirúrgico, a fim de evitar o crescimento de patógenos contaminantes. Na medicina veterinária, a antibioticoprofilaxia é claramente aceita como importante medida da redução e controle da incidência de infecções do sítio cirúrgico. Este estudo objetivou avaliar a utilização de antibióticos nos procedimentos cirúrgicos, realizados na clínica cirúrgica de cães e gatos do Hospital Veterinário da Universidade Federal de Viçosa, considerando a sua necessidade e a realidade. A população para o estudo foi constituída dos pacientes submetidos a tratamento cirúrgico, no período de 11 de maio a 11 de novembro de 2007. A utilização de antibióticos nos procedimentos estudados não foi padronizada ou alterada, para realização deste estudo. Assim, concluiu-se que é necessário estabelecer critérios para a antibioticoprofilaxia, bem como sua padronização no Hospital Veterinário da Universidade Federal de Viçosa, para corrigir possíveis falhas no processo de prevenção das infecções do sítio cirúrgico.
\end{abstract}

Palavras-chave: infecção, profilaxia, sítio cirúrgico.

\section{ABSTRACT}

\section{Antibiotic prophylaxis in surgery of dogs and cats: the necessity and the reality}

Antimicrobial prophylaxis aim at preventing surgical wound contamination after the exposure to any microorganism and before infection is established. Inhibitory antimicrobial concentrations should be reached and maintained on the incision throughout the entire surgical procedure in order to prevent microbial growth. In veterinary medicine, antimicrobial prophylaxis is clearly disclosure and accepted as an important action to control and reduce the incidence of surgical wound infection. This study evaluated the use of antibiotics in small animals' surgeries at the Veterinary Hospital of the Universidade Federal de Viçosa, comparing the need and the reality. The population in study consisted of patients undergoing surgical procedures from May 11 to November 11,2007. The routine use of antibiotics was not altered in any way for this study. The results showed that it is necessary to establish some criteria for the use of antimicrobials at the Veterinary Hospital of the Universidade Federal de Viçosa as well as the standardization of the same to correct possible failures in the process of surgical site infection.

Key words: infection, prophylaxis, surgical wound.

Recebido para publicação em 11/02/2011 e aprovado em 18/06/2012.

${ }^{1}$ Trabalho extraído da dissertação de mestrado do primeiro autor.

${ }^{2}$ Enfermeiro, Mestre. Departamento de Veterinária, Universidade Federal de Viçosa, Campus Viçosa, Avenida Peter Henry Rolfs, s/n, 36570-000, Viçosa, Minas Gerais, Brasil. danielpontesbraga@yahoo.com.br

${ }^{3}$ Médica Veterinária, Doutora. Departamento de Veterinária, Universidade Federal de Viçosa, Campus Viçosa, Avenida Peter Henry Rolfs, s/n, 36570-000, Viçosa, Minas Gerais, Brasil. Bolsista de produtividade do CNPq. andrea@ufv.br (autora para correspondência)

${ }^{4}$ Médica Veterinária, Mestre. Departamento de Veterinária, Universidade Federal de Viçosa, Campus Viçosa, Avenida Peter Henry Rolfs, s/n, 36570-000, Viçosa, Minas Gerais, Brasil. tatianabcarvalho@yahoo.com.br

${ }^{5}$ Médicas Veterinárias. Departamento de Veterinária, Universidade Federal de Viçosa, Campus Viçosa, Avenida Peter Henry Rolfs, s/n, 36570-000, Viçosa, Minas Gerais, Brasil. letícia.santos@ufv.br; camila.corsini@ufv.br 


\section{INTRODUÇÃO}

Na medicina veterinária, a administração profilática de antimicrobianos durante o procedimento cirúrgico é claramente aceita como importante medida da redução e controle da incidência de infecção do sítio cirúrgico (Roush, 1999; Whittem et al., 1999; Johnson, 2002; Hedlund, 2005; Siem $\&$ Fossum, 2005; Dunning, 2007). Entretanto, as recomendações e restrições são inúmeras e não divergem das empregadas na medicina humana. Ainda hoje, para a prevenção e redução das infecções do sítio cirúrgico, faz-se necessária a sua identificação, a sua mensuração e o seu controle, tema que ainda é, porém, polêmico e pouco abordado.

A antibioticoprofilaxia tem o objetivo de alcançar concentrações antimicrobianas inibitórias, no local da incisão, durante todo o procedimento cirúrgico, a fim de evitar o crescimento de patógenos contaminantes, no momento de uma potencial contaminação da ferida cirúrgica. De maneira geral, sua administração deve ser realizada entre 30 e 60 minutos antes da cirurgia (Roush, 1999; Whittem et al., 1999; Viana, 2001; Harari, 2004; Hedlund, 2005; Siem \& Fossum, 2005).

Vários autores apontaram a falta de padronização da antibioticoprofilaxia e seu uso indiscriminado como fatores potenciais da elevação da incidência de infecção, dos altos custos dos tratamentos, para a instituição e proprietários, e do risco crescente do surgimento de resistência antimicrobiana às drogas empregadas (Brown et al., 1997; Harari, 2004; Dunning, 2007). Ainda, a escolha do antimicrobiano a ser empregado deve ser baseada nos micro-organismos contaminantes que, sabidamente, têm maior probabilidade de causar infecção no sítio cirúrgico e que apresentem padrão de sensibilidade ao antibiótico escolhido (Roush, 1999; Siem \& Fossum, 2005; Dunning, 2007).

Couto et al. (1996) e Machado et al. (2001) recomendaram que a profilaxia antibiótica não deve ultrapassar o período da cirurgia, mesmo em situações de risco ou de contaminações acidentais, e que uma duração superior a 48 horas é inapropriada. Dunning (2007) e Siem \& Fossum (2005) indicaram a antibioticoprofilaxia, em conjunto com altos padrões de técnica asséptica, manipulação meticulosa e atraumática dos tecidos, hemostasia cuidadosa, uso criterioso de suturas, prevenção de suprimentos sanguíneo, eliminação de espaço morto, aproximação anatômica dos tecidos e cuidados pós-operatórios adequados.

Roush (1999) e Viana (2001) relataram que não há evidências científicas da redução da incidência de infecção com a continuação do tratamento com antibióticos por períodos mais longos que a cirurgia. Segundo Whittem et al. (1999), a administração pré-operatória, 30 minutos antes da cirurgia, sem a necessidade de administração de antibióticos após o procedimento, é capaz de reduzir efetivamente a taxa de infecção pós-operatória em cães submetidos à cirurgia ortopédica eletiva. A mesma indicação é feita por Soontornvipart et al. (2003) que verificaram a eficiência da antibioticoprofilaxia no pré-operatório de 60 cães com fraturas em ossos longos.

Johnson (2002) exemplificou a utilização de antimicrobianos não padronizada como uma das causas da resistência antimicrobiana e do aumento dos custos das intervenções médicas veterinárias, salientando que, apesar dos vários estudos no assunto, o uso de terapia antibiótica empírica (não padronizada) e profilática ainda é muito comum na medicina veterinária.

Dessa forma, o objetivo deste estudo foi avaliar a utilização de antibióticos nos procedimentos cirúrgicos realizados na Clínica Cirúrgica de Cães e Gatos do Hospital Veterinário da Universidade Federal de Viçosa, no período de 11 de maio a 11 de novembro de 2007, visando à sua padronização, e relacioná-la com o índice de infecção.

\section{MATERIAL E MÉTODOS}

O presente trabalho foi aprovado pela Comissão de Ética do Departamento de Veterinária da UFV, conforme o protocolo de número 68/2001.

A população para o estudo foi constituída dos pacientes submetidos a tratamento cirúrgico, na Clínica Cirúrgica de Cães e Gatos do Hospital Veterinário da Universidade Federal de Viçosa (UFV), no período de 11 de maio a 11 de novembro de 2007. Todos os animais submetidos aos procedimentos realizados pela clínica cirúrgica de cães e gatos foram eleitos como possíveis participantes deste estudo. No processo de seleção inicial, foram verificados todos os pacientes submetidos a procedimentos designados como cirúrgicos no período estabelecido.

A rotina dos pacientes submetidos a cirurgias não foi alterada. A maioria dos animais foi submetida a procedimento de tricotomia prévia na sala de preparação e, em seguida, encaminhada para a sala de cirurgia. Os procedimentos cirúrgicos analisados foram realizados na sala de cirurgia, onde ocorriam, normalmente, de uma a seis cirurgias por dia, sob a responsabilidade da mesma equipe.

A utilização de antibióticos nos procedimentos estudados não foi alterada para realização deste estudo. Dados referentes à utilização de antibioticoterapia ou profilaxia foram registrados, habitualmente, pelo cirurgião, na ficha clínica do paciente, durante o registro da cirurgia e, pelo anestesista, na ficha de anestesia, durante a realização da cirurgia, sendo mencionado o momento de sua utilização, nome comercial, fórmula química, dosagem e tempo de utilização. Também constaram, na ficha de evolução, a nota de progresso e a utilização de antimicrobiano (nome, dosagem e motivo).

Para classificação das cirurgias, adotaram-se os critérios de classificação internacional, propostos por Howard \& Culberson (1964) adotados pelo Ministério da Saúde na Portaria MS nº. 2.616 (Brasil, 1998; Roush, 1999; OMS, 2003; 
Harari, 2004; Brasil, 2005; Siem \& Fossum, 2005; Dunning, 2007), com os procedimentos sendo classificados pelo potencial de contaminação (grau de contaminação) da ferida cirúrgica, em cirurgia limpa, cirurgia potencialmente contaminada, cirurgia contaminada e cirurgia infectada.

A incidência de infecções do sítio cirúrgico (ISC) foi calculada de acordo com Brasil (2005), multiplicando-se o número de pacientes submetidos a cirurgias que apresentaram ISC por 100 e dividindo-se pelo número total de paciente submetidos a cirurgias.

\section{RESULTADOS E DISCUSSÃO}

No período estudado, a clínica cirúrgica de cães e gatos do Hospital Veterinário da UFV registrou 307 pacientes submetidos a procedimentos cirúrgicos.

Os resultados obtidos neste estudo revelaram que, dos 72 pacientes submetidos a cirurgias ortopédicas, 68 (94.4\%) receberam antibioticoprofilaxia. Foram detectados 12 casos de infecção do sítio cirúrgico (ISC) nesse grupo, sendo que todos pacientes receberam antibiótico profilático (Tabela 1). Esse fato pode indicar que a antibioticoprofilaxia, por si só, não é o fator determinante de ocorrência ou não de ISC, havendo outras causas a serem pesquisadas. Dunning (2007) apontou o uso profilático de antibióticos como um dos fatores para redução das infecções. Salientou, entretanto, que a antibioticoprofilaxia não deve substituir a técnica cirúrgica precisa, o planejamento pré-operatório e os cuidados pós-operatórios. Segundo Vilar-Compte et al. (2001), a utilização adequada de antibiótico como profilaxia está claramente associada à redução da incidência de infecção do sítio cirúrgico. Entretanto, neste estudo, observou-se que todos os casos ortopédicos de ISC receberam esse tratamento, o que provavelmente ocorreu pelo maior tempo cirúrgico e pela utilização de próteses.

Acredita-se que o uso inadequado de antibioticoprofilaxia é dos fatores de risco importantes para instalação da infecção hospitalar e do sítio cirúrgico. Sabe-se que a utilização abusiva de antibióticos, em dose e número de aplicações, está diretamente associada ao aumento da incidência de micro-organismos multirresistentes e ao aumento dos custos de internações (Brasil, 2000; Neto, 2003; Harari, 2004; Brasil, 2005; Siem \& Fossum, 2005; Dunning, 2007). Para suportar essa afirmação, caracterizou-se, por meio das Tabelas 2, 3 e 4, a utilização de antibioticoprofilaxia, considerando-se apenas os pacientes ortopédicos submetidos a intervenções cirúrgicas classificadas como limpas. Em tais cirurgias, a literatura aponta não haver a necessidade de utilização de antibioticoprofilaxia, pela baixa probabilidade de ocorrência de infecção (Siem \& Fossum, 2005; Dunning, 2007), demonstrando-se, assim, uma falta de sistematização, em que se percebe ausência de critério na utilização da antibioticoprofilaxia, o que pode sugerir uma possível falha no processo de prevenção das infecções do sítio cirúrgico.

Dos 56 pacientes submetidos a intervenções ortopédicas classificadas como limpas, foi verificada a ocorrência de ISC em oito casos. Destes, cinco pacientes receberam antibioticoprofilaxia apenas no pré-operatório; em um caso, no pré e pós-operatório; e, em dois outros, no pré, trans e pós-operatório (Tabelas 2, 3 e 4).

De um modo geral, verificou-se maior utilização de antibiótico profilático no pré e pós-operatório, em desacordo com as recomendações de Brasil (1998), Machado et al. (2001) e Oselka (2001). Notadamente a sistematização de seu uso de acordo com a classificação das cirurgias segundo seu potencial de contaminação e a duração inferior a 48 horas após o procedimento cirúrgico. Segundo Neto (2003) e Couto et al. (2003), a utilização de antimicrobianos em pacientes cirúrgicos fora da rotina de profilaxia pré-estabelecida e após a realização de procedimentos cirúrgicos limpos é um bom indicador de que o procedimento possa ter falhado no que se refere à prevenção das infecções hospitalares.

Ainda, notou-se falta de sistematização na escolha do antibiótico, bem como na periodicidade de aplicação, não se seguindo as orientações de Couto et al. (1996). É sabido que as infecções do sítio cirúrgico e a resistência bacteriana podem causar um grande impacto no futuro da medicina veterinária, prejudicando seriamente os tratamentos propostos e elevando os custos gerais dos tratamentos, com a perda do acesso a antibióticos efetivos, fato citado por Brown et al. (1997) e comprovado por Aiello et al. (2007).

Tabela 1. Ocorrência de infecção do sítio cirúrgico (ISC), segundo grau de contaminação da ferida operatória, em 72 pacientes submetidos a procedimentos ortopédicos, na clínica cirúrgica de cães e gatos do Hospital Veterinário da Universidade Federal de Viçosa, no período de 11 de maio a 11 de novembro de 2007 e a utilização de antibióticos durante os procedimentos

\begin{tabular}{|c|c|c|c|c|}
\hline \multirow{2}{*}{$\begin{array}{l}\text { Pacientes classificados por } \\
\text { potencial de contaminação }\end{array}$} & \multicolumn{2}{|c|}{$\begin{array}{c}\text { Utilização de antibiótico em algum } \\
\text { momento da cirurgia }\end{array}$} & \multicolumn{2}{|c|}{$\begin{array}{l}\text { Ocorrência de ISC e utilização de } \\
\text { antibiótico em algum momento da cirurgia }\end{array}$} \\
\hline & Sim & Não & Sim & Não \\
\hline Limpa & 53 & 3 & 8 & 0 \\
\hline Potencialmente contaminada & 8 & 0 & 2 & 0 \\
\hline Contaminada & 4 & 1 & 1 & 0 \\
\hline Infectada & 3 & 0 & 1 & 0 \\
\hline Total & 68 & 4 & 12 & 0 \\
\hline
\end{tabular}

Rev. Ceres, Viçosa, v. 59, n.6, p. 758-764, nov/dez, 2012 
Fazendo a mesma análise para pacientes submetidos a cirurgias obstétricas, no total de 108 pacientes, a Tabela 5 relaciona a incidência de ISC, segundo o grau de contaminação da ferida operatória e a utilização de antibióticos durante os procedimentos. As Tabelas 6, 7 e 8 referem-se aos pacientes submetidos a cirurgias obstétricas potencialmente contaminadas, para o que a literatura indica a utilização de antibioticoprofilaxia apenas em casos especiais. A Tabela 6 relaciona o momento cirúrgico de utilização de antimicrobianos e os casos de ISC, a Tabela 7 descreve os antibióticos utilizados e a Tabela 8 refere-se aos dois casos de ISC e a antibioticoprofilaxia utilizada.
Dentre os 77 pacientes submetidos a intervenções potencialmente contaminadas, verificaram-se a utilização de antibioticoprofilaxia em 76 pacientes e a ocorrência de ISC em dois casos nesse grupo (Tabelas 6 e 8). Um dos pacientes utilizou profilaxia no pré e pós-operatório e o outro no pós-operatório.

A Tabela 7 caracteriza claramente a utilização de antibioticoprofilaxia, principalmente no pós-operatório, e sua continuidade após 24 horas da cirurgia, na maioria dos pacientes. Isso contradiz as recomendações de Roush (1999), Viana (2001) e Dunning (2007), que desaconselharam a continuidade após 24 horas, e Couto et al. (1996) e

Tabela 2. Momento cirúrgico da utilização de antimicrobianos, total de pacientes em que o antimicrobiano foi utilizado naquele momento e ocorrência de infecção do sítio cirúrgico (ISC) em pacientes submetidos a cirurgias ortopédicas limpas na clínica cirúrgica de cães e gatos do Hospital Veterinário da Universidade Federal de Viçosa, no período de 11 de maio a 11 de novembro de 2007

\begin{tabular}{lccccc}
\hline Momento cirúrgico & Pacientes & Pré-operatório & Trans-operatório & Pós-operatório & Casos de ISC \\
\hline Nenhum momento & 3 & 0 & 0 & 0 & 0 \\
Somente no pré-operatório & 10 & 10 & 0 & 0 & 5 \\
Somente no trans-operatório & 2 & 0 & 2 & 0 & 0 \\
Somente no pós-operatório & 3 & 0 & 0 & 3 & 0 \\
Pré e pós-operatório & 31 & 31 & 0 & 31 & 0 \\
Pré e trans-operatório & 0 & 0 & 3 & 3 & 2 \\
Pré, trans e pós-operatório & 3 & 3 & 4 & 4 & 0 \\
Trans e pós-operatório & 4 & 0 & 9 & 41 & 8 \\
\hline Total de pacientes & 56 & 44 & & & \\
\hline
\end{tabular}

Tabela 3. Descrição detalhada dos antibióticos e momento da utilização nas cirurgias ortopédicas limpas de 53 pacientes, realizadas na clínica cirúrgica de cães e gatos do Hospital Veterinário da Universidade Federal de Viçosa, no período de 11 de maio a 11 de novembro de 2007

\begin{tabular}{|c|c|c|}
\hline Momento da cirurgia & Antibióticos e doses* utilizadas durante os procedimentos & $\begin{array}{c}\text { Pacientes em uso } \\
\text { de antibióticos }\end{array}$ \\
\hline \multirow{6}{*}{ Pré-operatório } & Cefalexina -1 dose & 35 \\
\hline & Cefalexina -1 dose / enrofloxacino -1 dose & 5 \\
\hline & Cefalexina -2 doses & 1 \\
\hline & Cefalexina - BID - 6 dias (12 doses) & 1 \\
\hline & Enrofloxacino -1 dose & 2 \\
\hline & Total & 44 \\
\hline \multirow{3}{*}{ Trans-operatório } & Cefalexina -1 dose & 8 \\
\hline & Cefalexina e enrofloxacino - 1 dose & 1 \\
\hline & Total & 9 \\
\hline \multirow{10}{*}{ Pós-operatório } & Ampicilina - 21 doses (TID), 7 dias & 1 \\
\hline & Cefalexina -1 dose & 1 \\
\hline & Cefalexina -7 doses (SID) 7 dias & 1 \\
\hline & Cefalexina $-8,15,16,21$ doses (BID), 4, 7, 8, 10 dias & 4 \\
\hline & Cefalexina - 14 doses (BID) 7 dias & 8 \\
\hline & Cefalexina -20 doses (BID) 10 dias & 21 \\
\hline & Ceftiofur -20 doses (BID) 10 dias & 1 \\
\hline & Doxiciclina -2 doses (SID) 2 dias & 1 \\
\hline & Enrofloxacino - 10 doses (SID) 10 dias & 3 \\
\hline & Total & 41 \\
\hline
\end{tabular}

SID (uma vez ao dia), BID (duas vezes ao dia) e TID (três vezes ao dia)

*Dose: é considerada dose cada aplicação indicada e prescrita pelo veterinário, sendo devidamente registrada na documentação clínica. 
Machado et al. (2001), que enfatizaram a profilaxia antibiótica no período da cirurgia, sendo duração superior a 48 horas considerada inapropriada.

Assim como verificado nas cirurgias ortopédicas limpas, observou-se, nos pacientes submetidos a cirurgias obstétricas potencialmente contaminadas uma falta de sistematização na utilização da antibioticoprofilaxia, com sua indicação contínua para o pós- operatório não estando também de acordo com as recomendações de Brasil (1998), Machado et al. (2001) e Oselka (2001).

$\mathrm{Na}$ análise dos registros clínicos referentes aos antibióticos profiláticos utilizados, verificou-se uma grande variedade de prescrições, no que se refere aos tipos, dosagens e periodicidade dos antibióticos empregados profilaticamente, dados esses que não encontram suporte na literatura pesquisada. Stehling et al. (2001), descre- vendo a prevenção e controle de infecções em ambiente cirúrgico veterinário, citaram a importância da antibioticoprofilaxia padronizada, o que também é indicado pela ANVISA (2000), Harari (2004) e Dunning (2007). A falta de padronização e de critérios para a antibioticoprofilaxia, observada neste estudo e demonstrada nas tabelas anteriores, contradiz as recomendações atualmente aceitas e pode ser um dos fatores que favoreceu a ocorrência de ISC, nas feridas classificadas como limpa ou potencialmente contaminadas.

Outro dado importante encontrado neste estudo foi que a anotação do antibiótico profilático, registrada pelo cirurgião responsável no prontuário clínico anterior ao ato cirúrgico, não foi encontrada. Observou-se que tal registro era geralmente feito pelo anestesista, no momento da cirurgia, na ficha de anestesia, evidenciando a admi-

Tabela 4. Descrição dos antibióticos utilizados por oito pacientes submetidos a cirurgias ortopédicas limpas, em que foi detectada infecção do sítio cirúrgico (ISC), realizadas na clínica cirúrgica de cães e gatos do Hospital Veterinário da Universidade Federal de Viçosa, no período de 11 de maio a 11 de novembro de 2007

\begin{tabular}{lcl}
\hline Momento cirúrgico & Pacientes & Antibióticos utilizados pelos pacientes que apresentaram ISC \\
\hline Pré-operatório & 5 & $\begin{array}{l}\text { cefalexina }-1 \text { dose }- \text { utilizada em } 3 \text { pacientes cefalexina }-1 \text { dose } / \\
\text { enrofloxacino }-1 \text { dose }- \text { utilizados em } 2 \text { pacientes } \\
\text { cefalexina }-1 \text { dose }+ \text { ceftiofur BID }-10 \text { dias }(2 \text { x } 10=20 \text { doses }) \\
\text { Pré e pós-operatório }\end{array}$ cefalexina -1 dose (no pré e transoperatório + cefalexina BID - $_{07 \text { e } 10 \text { dias })(14 \text { e } 21 \text { doses })}$ \\
Pré, trans e pós-operatório & 2 &
\end{tabular}

Tabela 5. Incidência de infecção do sítio cirúrgico (ISC) segundo grau de contaminação da ferida operatória em 108 pacientes submetidos a procedimentos obstétricos na clínica cirúrgica de cães e gatos do Hospital Veterinário da Universidade Federal de Viçosa, no período de 11 de maio a 11 de novembro de 2007 e a utilização de antibióticos durante os procedimentos

\begin{tabular}{|c|c|c|c|c|}
\hline \multirow{2}{*}{$\begin{array}{l}\text { Pacientes classificados por } \\
\text { potencial de contaminação }\end{array}$} & \multicolumn{2}{|c|}{$\begin{array}{c}\text { Utilização de antibiótico em algum } \\
\text { momento da cirurgia }\end{array}$} & \multicolumn{2}{|c|}{$\begin{array}{l}\text { Ocorrência de ISC e utilização de } \\
\text { antibiótico em algum momento da cirurgia }\end{array}$} \\
\hline & Sim & Não & Sim & Não \\
\hline Limpa & 0 & 0 & 0 & 0 \\
\hline Potencialmente contaminada & 76 & 1 & 2 & 0 \\
\hline Contaminada & 21 & 0 & 1 & 0 \\
\hline Infectada & 10 & 0 & 1 & 0 \\
\hline Total & 107 & 1 & 4 & 0 \\
\hline
\end{tabular}

Tabela 6. Momento cirúrgico, utilização de antimicrobianos e infecção do sítio cirúrgico (ISC) em 77 pacientes submetidos a cirurgias obstétricas potencialmente contaminadas na clínica cirúrgica de cães e gatos do Hospital Veterinário da Universidade Federal de Viçosa, no período de 11 de maio a 11 de novembro de 2007

\begin{tabular}{lccccc}
\hline Momento cirúrgico & Pacientes & Pré-operatório & Trans-operatório & Pós-operatório & ISC \\
\hline Nenhum momento & 1 & 0 & 0 & 0 & 0 \\
Somente no pré-operatório & 21 & 21 & 0 & 0 & 0 \\
Somente no trans-operatório & 5 & 0 & 5 & 0 & 0 \\
Somente no pós-operatório & 13 & 0 & 0 & 13 & 1 \\
Pré e pós-operatório & 23 & 23 & 1 & 23 & 1 \\
Pré e trans-operatório & 1 & 1 & 2 & 2 & 0 \\
Pré, trans e pós-operatório & 2 & 2 & 11 & 11 & 0 \\
Trans e pós-operatório & 11 & 0 & 19 & 49 & 2 \\
\hline Total de pacientes & 77 & 47 & & \\
\hline
\end{tabular}

Rev. Ceres, Viçosa, v. 59, n.6, p. 758-764, nov/dez, 2012 
nistração da antibioticoprofilaxia nos momentos iniciais da cirurgia, o que difere das recomendações de Brown et al. (1997), Whittem et al. (1999), Hedlund (2005) e Siem \& Fossum (2005), discutidas anteriormente.

Também, o registro da continuação da antibioticoprofilaxia, utilizando esquemas variados de doses e dias de tratamento, geralmente se fez presente e completo nas fichas cirúrgicas e no prontuário clínico, sendo que esta continuidade não é indicada na literatura (Couto et al., 1996; Roush, 1999; Machado et al., 2001; Viana, 2001; Dunning, 2007).

Estes fatos levam a crer que a utilização de antimicrobianos profilaticamente em cirurgias ortopédicas e obstétricas mostrou-se inadequada às linhas empregadas e indicadas atualmente, visto que, aparentemente, não foram capazes de evitar a ISC, já que todos os pacientes submetidos a cirurgias ortopédicas limpas e obstétricas potencialmente contaminadas que apresentaram ISC receberam antibioticoprofilaxia.

Os resultados verificados sugerem a necessidade de padronização da antibioticoprofilaxia, para sua adequada utilização, associada ao controle de outros fatores de risco, principalmente nas cirurgias limpas e potencialmente contaminadas, de acordo com literatura específica. Para tal, uma mudança de postura seria necessária para alterar esse quadro, com uma possível redução dos índices de ISC, como, por exemplo, duração.

Segundo Soontornvipart et al. (2003) e Smith (2004), para a prevenção e o controle das ISC é preciso um programa de controle eficiente com base no conhecimento epidemiológico, com estudo e monitoramento dos microorganismos presentes, da resistência antimicrobiana e a correta utilização da medicação profilática a ser empregada.

Tabela 7. Descrição detalhada dos antibióticos e momento de sua utilização nas cirurgias obstétricas potencialmente contaminadas de 76 pacientes realizadas na clínica cirúrgica de cães e gatos do Hospital Veterinário da Universidade Federal de Viçosa, no período de 11 de maio a 11 de novembro de 2007

\begin{tabular}{|c|c|c|}
\hline Momento da cirurgia & Antibióticos e doses utilizadas durante os procedimentos & ade de pacientes \\
\hline \multirow{7}{*}{ Pré-operatório } & Amoxicilina + clavulanato -1 dose & 1 \\
\hline & Ampicilina - 1 dose & 1 \\
\hline & Doxiciclina -4 doses & 1 \\
\hline & Enrofloxacino -1 dose & 38 \\
\hline & Enrofloxacino - 1 dose / ampicilina - 1 dose & 5 \\
\hline & Enrofloxacino - 1 dose / enrofloxacino SID - 2 dias ( 2 doses) & 1 \\
\hline & Total & 47 \\
\hline \multirow{4}{*}{ Trans-operatório } & Ampicilina - 1 dose & 1 \\
\hline & Enrofloxacino - 1 dose / ampicilina - 1 dose & 1 \\
\hline & Enrofloxacino -1 dose & 17 \\
\hline & Total & 19 \\
\hline \multirow{12}{*}{ Pós-operatório } & Amoxicilina BID - 7 dias (14 doses) & 1 \\
\hline & Amoxicilina + clavulanato BID - 10 dias (20 doses) & 1 \\
\hline & Enrofloxacino -1 dose & 7 \\
\hline & Enrofloxacino - 1 dose / ampicilina - 1 dose & 1 \\
\hline & Enrofloxacino BID - 10 dias (20 doses) & 1 \\
\hline & Enrofloxacino BID - 5 e 7 dias ( 10 e 14 doses) & 3 \\
\hline & Enrofloxacino SID - 10 dias (10 doses) / ampicilina TID 10 dias (30 doses) & 4 \\
\hline & Enrofloxacino SID - 10 dias (10 doses) / ampicilina QID 10 dias (40 doses) & 2 \\
\hline & Enrofloxacino - 1 dose / enrofloxacino SID - 7 dias ( 7 doses) & 5 \\
\hline & Enrofloxacino SID - 3, 5, 7 e 10 dias (3, 5, 7 e 10 doses) & 22 \\
\hline & Enrofloxacino SID - 7 dias (7 doses) / ampicilina TID 7 dias (21 doses) & 2 \\
\hline & Total & 49 \\
\hline
\end{tabular}

SID (uma vez ao dia), BID (duas vezes ao dia), TID (três vezes ao dia) e QID (quatro vezes ao dia).

Tabela 8. Descrição dos antibióticos utilizados em dois pacientes submetidos à cirurgia obstétrica potencialmente contaminadas, que apresentaram infecção do sítio cirúrgico (ISC), realizada na clínica cirúrgica de cães e gatos do Hospital Veterinário da Universidade Federal de Viçosa, no período de 11 de maio a 11 de novembro de 2007

\begin{tabular}{lcl}
\hline Momento cirúrgico & Pacientes & Antibióticos utilizados pelos pacientes que apresentaram ISC \\
\hline Pré e pós-operatório & 1 & Enrofloxacino -1 dose / Enrofloxacino SID - 7 dias (7 doses) \\
\hline Pós-operatório & 1 & Enrofloxacino -1 dose \\
\hline
\end{tabular}




\section{CONCLUSÕES}

Com base nos resultados obtidos e nas condições da presente pesquisa conclui-se que a antibioticoprofilaxia, por si só, não é o fator determinante de ocorrência ou não de infecção do sítio cirúrgico, havendo outras causas a serem pesquisadas.

Não há padronização e critérios na antibioticoprofilaxia, no Hospital Veterinário da Universidade Federal de Viçosa, o que pode sugerir uma possível falha no processo de prevenção das infecções do sítio cirúrgico.

\section{REFERÊNCIAS}

ANVISA - Agência Nacional de Vigilância Sanitária (2000) Epidemiologia para o controle de infecção hospitalar. Brasília, ANVISA. 177p. (Caderno A).

Aiello G, Battaglia L, Arias MV \& Freitas JC (2007) Determinação dos índices de infecção hospitalar em um centro cirúrgico universitário veterinário de pequenos animais. Acta Science Veterinary, 35:345-346.

Brasil - Ministério da Saúde (1998) Aprova programa de controle de infecção hospitalar e dá outras providências. Portaria $\mathrm{n}^{\circ}$ 2.616, de 12 de maio de 1998. Brasília, Diário Oficial da União. 13 de maio de 1998, Seção 1, p.133-5.

Brasil - Ministério da Saúde (2000) Agência Nacional de Vigilância Sanitária (ANVISA). Aprova o roteiro de inspeção do programa de controle de infecção hospitalar. Resolução RDC n ${ }^{\circ} 48$, de 2 de junho de 2000. Brasília, Diário Oficial da União. 6 de junho 2000. Disponível em: 〈http://e-legis.anvisa.gov.br/leisref>. Acessado em: 8 de março de 2006.

Brasil - Ministério da Saúde (2005) Pediatria: prevenção e controle de infecção hospitalar. Brasília, Ministério da Saúde. 116p. (Série A - Normas e manuais técnicos).

Brown DC, Conzemius MG, Shofer F \& Swann H (1997) Epidemiologic evaluation of postoperative wound infections in dogs and cats. Journal of the American Veterinary Medical Association, 210:1302-1306.

Couto P, Magalhães ACG, Osório L \& Chambriard C (1996) Infecção hospitalar: critérios para instalação e atuação da comissão responsável, medidas profiláticas e emprego da antibioticoterapia. Revista Brasileira de Ortopedia, 31:862-866.

Couto RC, Pedrosa TM \& Nogueira JM (2003) Infecção hospitalar e outras complicações não-infecciosas da doença, epidemiologia, controle e tratamento. $3^{\mathrm{a}}$ ed. São Paulo, Medsi. 904p.

Dunning D (2007) Infecção da ferida cirúrgica e uso de antimicrobianos. In: Slatter D (Ed.) Manual de cirurgias de pequenos animais. São Paulo, Manole. p.113-122.

Harari J (2004) Controle de infecção. In: Harari J (Ed.) Segredos em cirurgias de pequenos animais. São Paulo, Artmed. p.25-32.

Hedlund C S (2005) Cirurgia do sistema tegumentar. In: Fossum TW (Ed.) Cirurgia de pequenos animais. São Paulo, Roca. p.71-80.

Howard JM \& Culberson WR (1964) Post-operative wound infections: the influence of ultraviolet irradiation of the operating room and of various others factors. Annals of Surgery, 160:1-192.

Johnson JA (2002) Nosocomial infections. Veterinary Clinics of North America: Small Animal Practice, 32:1101-1126.

Machado A, Ferraz AAB, Ferraz E, Arruda E, Nobre J, Konkewicz LR, Pimentel ML, Leão MTC, Trabasso P \& Grimbaum R (2001) Projeto diretrizes. São Paulo, Associação Médica e Conselho Federal de Medicina. 32p.
Neto MC (2003) Bactérias multirresistentes. In: Couto RC, Tânia MGP \& Débora BA (Eds.) Infecção hospitalar e outras complicações não-infecciosas da doença: epidemiologia, controle e tratamento. Belo Horizonte, Medsi. p.579-588.

OMS - Organización Mundial De La Salud (2003) Prevención de las infecciones nosocomiales: Guía práctica. $2^{\mathrm{a}}$ ed. Malta, OMS. 65p.

Oselka G (2001) A prescrição de antibióticos e as comissões de controle de infecção hospitalar. Revista da Associação Médica Brasileira, 47:102.

Roush JK (1999) Controle de infecção. In: Harari J (Ed.) Cirurgias de pequenos animais. Porto Alegre, Artmed. 420p.

Siem HB \& Fossum TW (2005) Infecções cirúrgicas e seleção antibiótica. In: Fossum TW (Ed.) Cirurgia de pequenos animais. São Paulo, Roca. p.61-70.

Smith BP (2004) Evolution of equine infection control programs. Veterinary Clinics of North America: Equine Practice, 20:521-30

Soontornvipart K, Neaas A, Dvofiak MJ \& Zatloukal J (2003) Smola: posttraumatic bacterial infections in extremities before and after osteosynthesis in small animals. Acta Veterinaria Brunensis, 72:249-260.

Stehling MC, Cunha AF \& Maria E (2001) Prevenção e controle de infecção em serviço de medicina veterinária. In: Martins MA (Ed.) Manual de infecção hospitalar. Epidemiologia, prevenção e controle. Belo Horizonte, Medice. p.915-927.

Viana FAB (2001) Abordagem da infecção em medicina veterinária - Uso de antimi-crobianos. In: Martins MA (Ed.) Manual de infecção hospitalar. Epidemiologia, prevenção e controle. Belo Horizonte, Medice. p.928-952.

Vilar-Compte D, Roldan R, Sandoval S, Coraminas R, De La Rosa M, Gordillo P \& Volkow P (2001) Surgical site infections in ambulatory surgery: a 5-year experience. American Journal of Infection Control, 29:99-103.

Whittem TL, Johnson AL, Smith CW, Schaeffer DJ, Coolman BR, Averill SM, Cooper TK, Merkin, GR. (1999) Effect of perioperative prophylactic antimicrobial treatment in dogs undergoing elective orthopedic surgery. Journal of American Medical Association, 215:212-216. 